\title{
Formation of epitaxial MnBi layers on (Ga,Mn)As
}

\author{
J. Adell, ${ }^{1}$ M. Adell, ${ }^{1}$ I. Ulfat, ${ }^{1}$ L. Ilver, ${ }^{1}$ J. Sadowski ${ }^{2,3}$ and J. Kanski ${ }^{1}$ \\ ${ }^{1}$ Department of Applied Physics, Chalmers University of Technology, SE-412 96 Göteborg, Sweden \\ ${ }^{2}$ MAX-lab, Lund University, SE-221 00 Lund, Sweden \\ ${ }^{3}$ Institute of Physics, Polish Academy of Sciences, aleja Lotnikow 32/46, 02-668 Warszawa, Poland \\ (Received 5 March 2009; revised manuscript received 3 July 2009; published 10 August 2009)
}

\begin{abstract}
The initial growth of MnBi on MnAs-terminated (GaMn)As is studied by means of synchrotron-based photoelectron spectroscopy. From analysis of surface core-level shifts we conclude that a continued epitaxial $\mathrm{MnBi}$ layer is formed, in which the $\mathrm{MnAs} / \mathrm{MnBi}$ interface occurs between $\mathrm{As}$ and $\mathrm{Bi}$ atomic planes. The well-defined $1 \times 2$ surface reconstruction of the MnAs surface is preserved for up to $2 \mathrm{ML}$ of MnBi before clear surface degradation occurs. The MnBi layer appears to be free from intermixed As.
\end{abstract}

DOI: $10.1103 /$ PhysRevB.80.075204

PACS number(s): 71.55.Eq, 75.50.Pp

\section{INTRODUCTION}

The interest in diluted magnetic semiconductors remains high and (Ga,Mn)As continues to play the important role of a model system for investigation of electronic and magnetic properties. ${ }^{1,2}$ For some time it was believed that the Curie temperature $\left(T_{\mathrm{C}}\right)$ of this system would be limited to around $110 \mathrm{~K}$ for fundamental (but unknown) reasons. Eventually the negative role of point defects, especially Mn atoms in interstitial sites, was recognized, and it was found that these particular defects could be removed by post-growth annealing. Thus, several groups have been able to produce (Ga,Mn)As layers with ferromagnetic transition temperatures in the range $160-185 \mathrm{~K}^{3,4}$ The limiting mechanisms are still not fully understood. According to mean-field calculations, ${ }^{5}$ which have been successfully applied for low Mn concentrations, the Curie temperature is proportional to the density of uncompensated magnetic moments. Within this model roomtemperature ferromagnetism is predicted for efficient $\mathrm{Mn}$ concentrations above $10 \%$. However, although very efficient ways to remove the compensating $\mathrm{Mn}$ have been developed, ${ }^{3,4}$ recent efforts in this direction ${ }^{6,7}$ have not been successful even with post-growth annealed samples with above $20 \% \mathrm{Mn}$. It is thus possible that antiferromagnetic superexchange coupling between neighbor substitutional Mn sites becomes a fundamental hinder to reach higher $T_{\mathrm{C}}: s$.

An alternative, still unexplored approach might be to integrate epitaxial layers of ferromagnetic materials, e.g., MnAs, MnSb, or MnBi in, e.g., (GaMn)As. Calculations ${ }^{8}$ predict that in the bulk form these materials should exhibit half-metallic ferromagnetism in the zincblende structure and $\mathrm{MnBi}$ is expected to remain a ferromagnetic half metal in the tetragonally strained configuration expected for an epitaxial layer on GaAs. ${ }^{9}$ We have demonstrated earlier ${ }^{10}$ that epitaxial 1-2 monolayers thick MnAs is obtained by post-growth annealing of (GaMn)As under As capping and that such layers can indeed be integrated epitaxially in (GaMn)As. The purpose of the present study is to explore whether similar epitaxial MnBi layers can be formed on (GaMn)As. We use core-level photoemission to follow the MnBi layer formation as we deposit individual atomic layers of $\mathrm{Bi}$ and $\mathrm{Mn}$.

Previous work on $\mathrm{MnBi}$ has been largely motivated by its unusual magnetic and magneto-optic properties. ${ }^{11} \mathrm{MnBi}$ crystallizes in NiAs-type hexagonal structure and the magnetocrystalline anisotropy directs the easy magnetization along the $c$-axis. MnBi films are commonly prepared by sequential deposition of $\mathrm{Bi}$ and $\mathrm{Mn}$ layers and annealing ${ }^{12}$ around $150-300{ }^{\circ} \mathrm{C}$. Such films are usually polycrystalline, but with high degree of $c$-axis oriented texture, which makes them particularly interesting for magnetic memory applications.

$\mathrm{MnBi}$ in zinc-blende structure has not been synthesized previously, although, as already mentioned, theoretical studies emphasize its potential use in spintronics applications due to the predicted half-metallic ferromagnetic state. To the extent that extremely thin films like those discussed here are ferromagnetic (this remains to be investigated), the magnetic anisotropy should give in-plane magnetization.

Another point of concern in the present study is the possibility of As/Bi intermixing. Since the spin-orbit interaction is significantly smaller in MnAs than in $\mathrm{MnBi},{ }^{13}$ As intermixing could modify the magnetic properties of the layers. This question is addressed here by detailed analysis of the As3d spectra at different stages of MnBi layer formation.

\section{EXPERIMENT}

The experiments were performed at beamline 41 at the Swedish national synchrotron-radiation laboratory MAX-lab, where a molecular beam epitaxy (MBE) system is connected to the photoelectron spectrometer for on-line sample preparation. The samples were grown on $1 \times 1 \mathrm{~cm}^{2}$ pieces of $n$-type epiready GaAs(100) wafers, which were glued by In to Mo sample holders. The substrate temperature was measured with an IR pyrometer and the Ga and Mn beam fluxes were calibrated by means of reflection high-energy electron diffraction (RHEED) oscillations. ${ }^{14}$ After conventional oxide desorption and growth of a GaAs buffer at $580{ }^{\circ} \mathrm{C}$, the substrate temperature was reduced to $230{ }^{\circ} \mathrm{C}$ for growth of a $500 \AA$ thick $\mathrm{Ga}_{0.95} \mathrm{Mn}_{0.05}$ As layer. The surface of this asgrown layer showed a weak but clear $1 \times 2$ reconstruction. After annealing at $230{ }^{\circ} \mathrm{C}$ under As capping the half-order diffraction streaks were significantly enhanced. Before the following $\mathrm{Bi}$ and $\mathrm{Mn}$ deposition the As source was turned off and the vacuum in the MBE chamber was reduced into the 
$10^{-9}$ torr range in order to minimize risks of inclusion in the MnBi film.

$\mathrm{Bi}$ was deposited on this surface at room temperature (RT). Monolayer coverage was estimated to be reached when the RHEED pattern was vanishing. Subsequent core-level analysis indicated that the dosing was somewhat in excess of one monolayer. A clear $1 \times 2$ RHEED pattern was restored after a new annealing treatment for $90 \mathrm{~min}$ at $195{ }^{\circ} \mathrm{C}$. On this surface additional monolayers of $\mathrm{Mn}$ and $\mathrm{Bi}$ were deposited sequentially, $\mathrm{Mn}$ at $200{ }^{\circ} \mathrm{C}$ and $\mathrm{Bi}$ at RT followed by 90 min annealing at $195{ }^{\circ} \mathrm{C}$. In this way altogether three $\mathrm{Bi}$ and two Mn layers were deposited.

Angle resolved photoemission from the shallow core levels of $\mathrm{As}$ and $\mathrm{Bi}$ was used to follow the development of adsorbed layers. The photoemission spectra were recorded in the plane of light incidence with the $p$-polarized light incident at $45^{\circ}$ relative surface normal. It is important to stress that the samples were transferred between the growth and analysis chambers under UHV conditions. The direct connection of the two systems allows us to follow the sample development after sequential growth steps. Most significantly, it allows us to study well defined as-grown samples without any need to restore the surface. As the material is metastable and undergoes phase separation around $300{ }^{\circ} \mathrm{C}$, its surface cannot be restored to a well-ordered state once it has been exposed to air.

\section{RESULTS AND DISCUSSION}

The overlayers were deposited in the MBE system and the surfaces were monitored during growth by means of RHEED. Although some degradation could be noticed after the third $\mathrm{Mn}$ and $\mathrm{Bi}$ deposition cycle, up to that point a streaky and low background $1 \times 2$ pattern was observed, typical for a flat and well-ordered surface. After transfer to the spectrometer chamber the surface order was confirmed by low-energy electron diffraction.

A continuous layer growth could also be concluded from the vacuum level cut off in the photoemission data. In Fig. 1 we show the development of the low-energy range of the spectra, which are displayed on a relative energy scale with reference to the initial MnAs-terminated situation (a). After the first $\mathrm{Bi}$ deposition the cut off is lowered by $0.50 \mathrm{eV}$ [spectrum (b)]. Apart from the shift, it is noted that the spectrum does not show any secondary structure at the position of the initial cut off. We can, therefore, conclude that the surface is essentially free from uncovered patches and that the streaky RHEED pattern of this surface does indeed represent the $\mathrm{Bi}$ covered surface and not uncovered regions of the initial MnAs-terminated (Ga,Mn)As surface. After subsequent deposition of a monolayer $\mathrm{Mn}$ the vacuum cut-off shifts further by $0.25 \mathrm{eV}$ [spectrum (c)] and another layer of $\mathrm{Bi}$ gives an additional shift of $0.07 \mathrm{eV}[\operatorname{spectrum}(\mathrm{d})]$. In the following deposition cycle of $\mathrm{Mn}$ and $\mathrm{Bi}$ monolayers the cut-off returns to positions $\mathrm{c}$ and $\mathrm{d}$, respectively.

We note that spectrum (d) does not show the typical monotonous decay toward increasing energy but has a broad structure in the range of the cut off of the Bi-terminated MnAs surface. We interpret this structure as a secondary cut

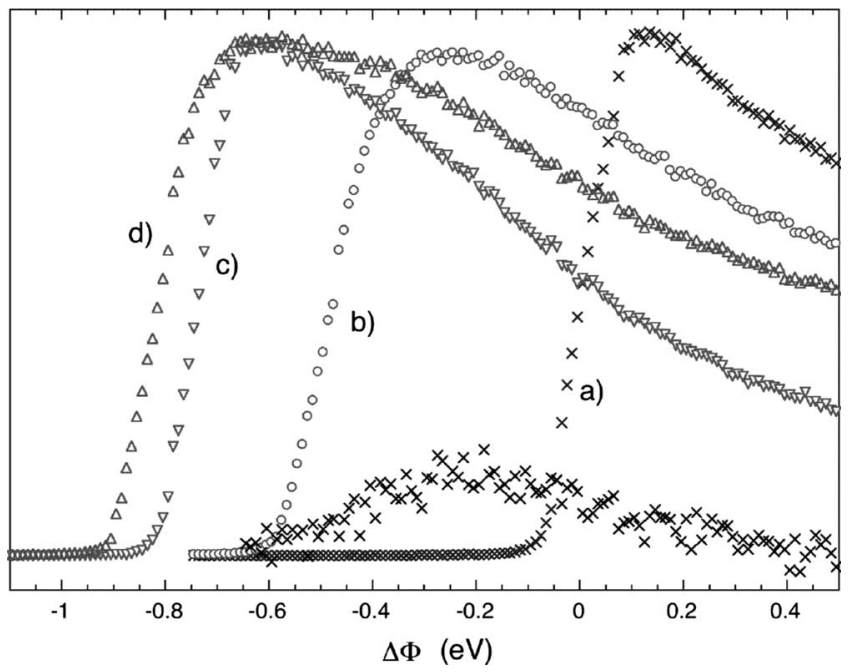

FIG. 1. Development of the low-energy cut-off range with Bi and Mn adsorption. (a) MnAs-terminated GaMnAs, (b) after deposition of $1 \mathrm{ML} \mathrm{Bi}$ and annealing, (c) after adsorption of 1 ML Mn, and (d) after a second adsorption of Bi and annealing.

off due to surface regions covered with excess Bi. By subtracting spectrum (b) from spectrum (d) (after adequate shift) we find that the difference, shown by small symbols in Fig. 1 , has a low-energy limit rather close to spectrum (b). The two systems are apparently terminated in similar ways, with a double layer of column $\mathrm{V}$ elements on top of a Mn layer. Thus, the data indicate that $\mathrm{Bi}$ adsorbed on the MnAsterminated surface does not break up the Mn-As bonds but forms an ordered layer on top of the As atoms.

The work-function changes reflect modifications of the surface dipole, i.e., charge redistributions between the outermost surface planes. Using the most common electronegativity scale of Pauling, according to which the electronegativities of As, Bi, and Mn are, respectively, 2.18, 2.02, and 1.55, one should thus expect a slightly reduced work function after the first Bi adsorption. Therefore it is somewhat surprising to see that the work function of the Bi-terminated surface is reduced by as much as $0.5 \mathrm{eV}$, and that the following reduction after $\mathrm{Mn}$ adsorption is smaller $(0.25 \mathrm{eV})$ despite the clearly lower electronegativity of $\mathrm{Mn}$. One obvious reason for this result is that the surface dipole depends not only on the last two atomic planes. In the present case the As atoms in the MnAs layer are polarized before the $\mathrm{Bi}$ adsorption due to interaction with $\mathrm{Mn}$, and therefore the atomic electronegativity of As is not an appropriate parameter. The relatively small and reversible changes found after the last $\mathrm{Mn}$ and $\mathrm{Bi}$ depositions suggest that the effective electronegativity difference between $\mathrm{Bi}$ and $\mathrm{Mn}$ is relatively small. On alternative electronegativity scales that take into account the atomic size (Allred Rochow and Sanderson scales) the electronegativities of $\mathrm{Bi}$ (1.67 and 2.34) are indeed much closer to those of Mn (1.60 and 2.20) than to As (2.20 and 2.82), though in all cases $\mathrm{Bi}$ is somewhat more negative. Nevertheless, in all cases $\mathrm{Mn}$ is less electronegative than $\mathrm{Bi}$, which should give surface dipoles of opposite signs than those found here if only the two last layers were active. A more detailed quantitative analysis of the charge distribution would be needed to 


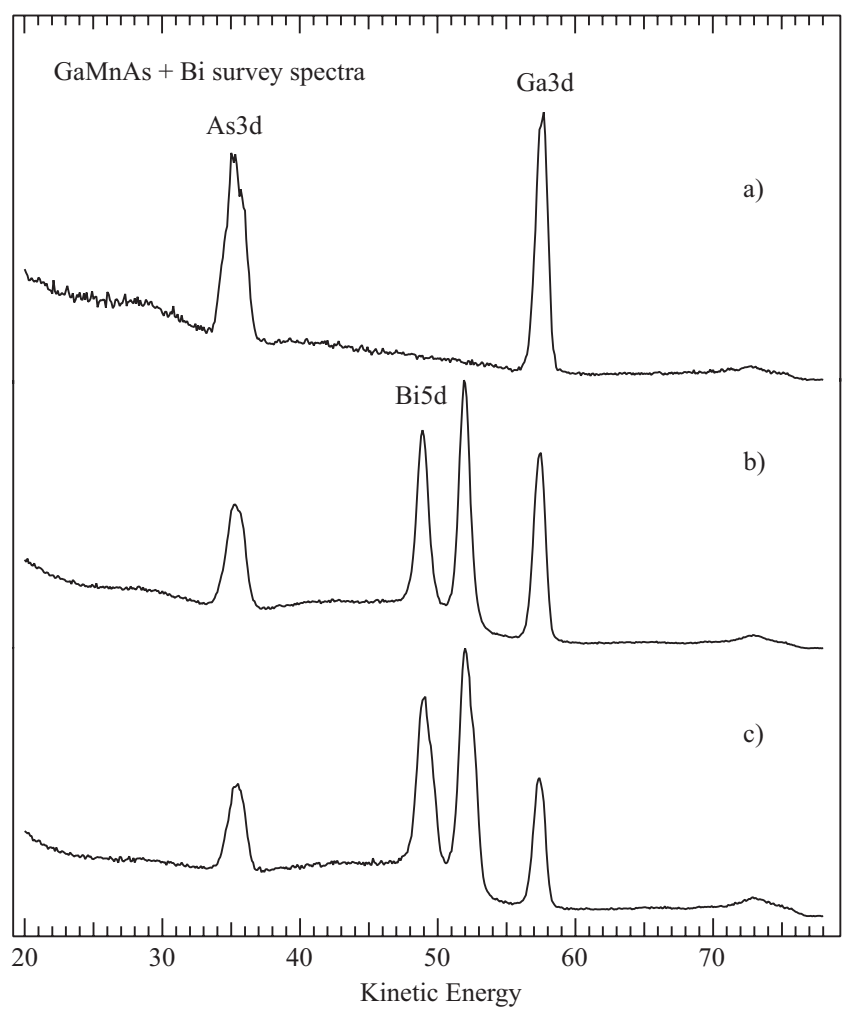

FIG. 2. Survey spectra obtained in normal emission excited with $81 \mathrm{eV}$ photons. (a) The starting MnAs-terminated surface, (b) after 1 ML Bi deposition and annealing, and (c) after the second deposition and annealing [corresponding to spectrum (d) in Fig. 1].

describe the work-function changes observed here. Such analysis is beyond the scope of this work.

The formation of the MnBi overlayer can also be followed qualitatively by the development of photoemission survey spectra, see Fig. 2. After the first Bi deposition and annealing at $195{ }^{\circ} \mathrm{C}$ [spectrum (b)] the As/Ga intensity ratio is reduced due to the removal of loosely adsorbed surface As. With a Mn layer adsorption and a second $\mathrm{Bi}$ deposition and annealing [spectrum (c)] both $\mathrm{Ga}$ and $\mathrm{As}$ peaks are reduced relative to the $\mathrm{Bi}$ and $\mathrm{Mn}$ emission. The apparently stronger attenuation of the $\mathrm{Ga}$ peak is explained by the shorter elastic mean-free path in the kinetic energy range 50-60 eV than around $35 \mathrm{eV}$. Thus, the As and Ga signals decrease systematically as we add monolayers of Bi and Mn, just as expected from layer-by-layer coverage.

To get a more detailed picture of the overlayer character we analyze the As3d and Bi5d core-level spectra. We start with As, which is the more complicated case due to a number of strongly overlapping components. The spectral decomposition presented in Fig. 3 is based on requirements of consistency between spectra obtained at different emission angles and under different surface conditions. In addition, we have also made use of a recent analysis of spectra from Asannealed $(\mathrm{Ga}, \mathrm{Mn}) \mathrm{As}$, in which we were eventually able to identify the component representing the MnAs surface layer. ${ }^{15}$ The fitting parameters are summarized in Table I. We find that a consistent decomposition of the spectrum obtained from a sample annealed under As can be achieved with five components, which we assign as atoms in the (Ga,Mn)As

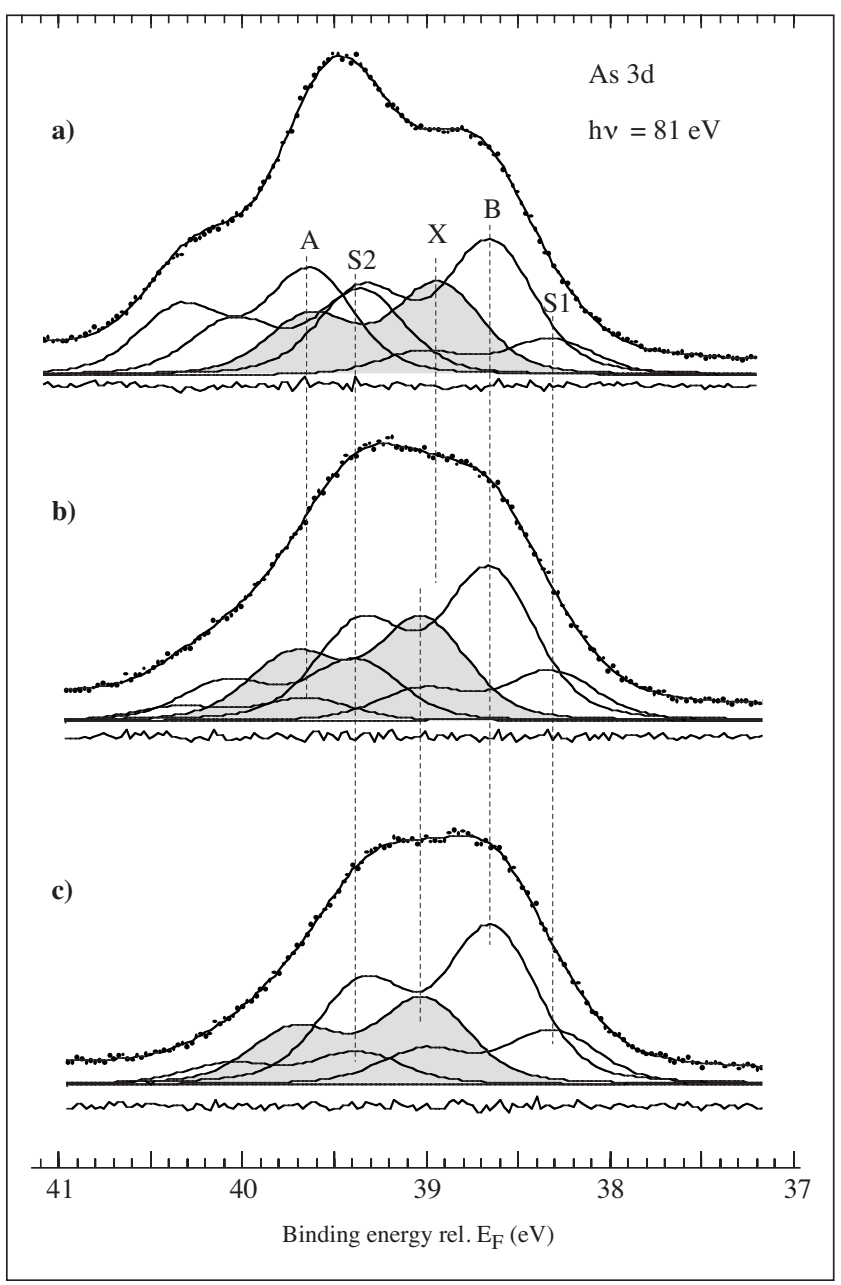

FIG. 3. Normal emission As3d spectra. (a) The initial MnAsterminated surface, (b) after Bi deposition and annealing, and (c) after additional $\mathrm{Mn}$ and $\mathrm{Bi}$ deposition and annealing.

bulk (B), adsorbed As (A), two surface components (S1, S2) and a fifth component $(\mathrm{X})$ which derives from the reacted MnAs layer. ${ }^{15}$ The spectrum strongly resembles that from as-grown (Ga,Mn)As, ${ }^{16}$ though a direct comparison shows immediately that the spectra differ with respect to their total widths. By comparing the angular and thermal dependences we can find the corresponding components in the two spectra. In particular we find that component X (see Fig. 3) is more stable with respect to annealing than any of the components found on the as-grown surface (except for the bulk component). The increased width of the spectrum from the MnAs terminated is caused by a clearly larger separation

TABLE I. Parameters used for fitting of As $3 \mathrm{~d}$ and $\mathrm{Bi}_{5 / 2}$ in Figs. 3-5, respectively.

\begin{tabular}{ccc}
\hline \hline & As3d & Bi5d $_{5 / 2}$ \\
\hline$\Delta \mathrm{E}_{\text {s-o }}$ & $0.695 \mathrm{eV}$ & \\
Branching ratio & 1.69 & \\
$\Delta \mathrm{E}_{\text {Gauss }}$ & $0.46 \mathrm{eV}$ & $0.63 \mathrm{eV}$ \\
$\Delta \mathrm{E}_{\text {Lorentz }}$ & $0.17 \mathrm{eV}$ & $0.27 \mathrm{eV}$ \\
\hline \hline
\end{tabular}



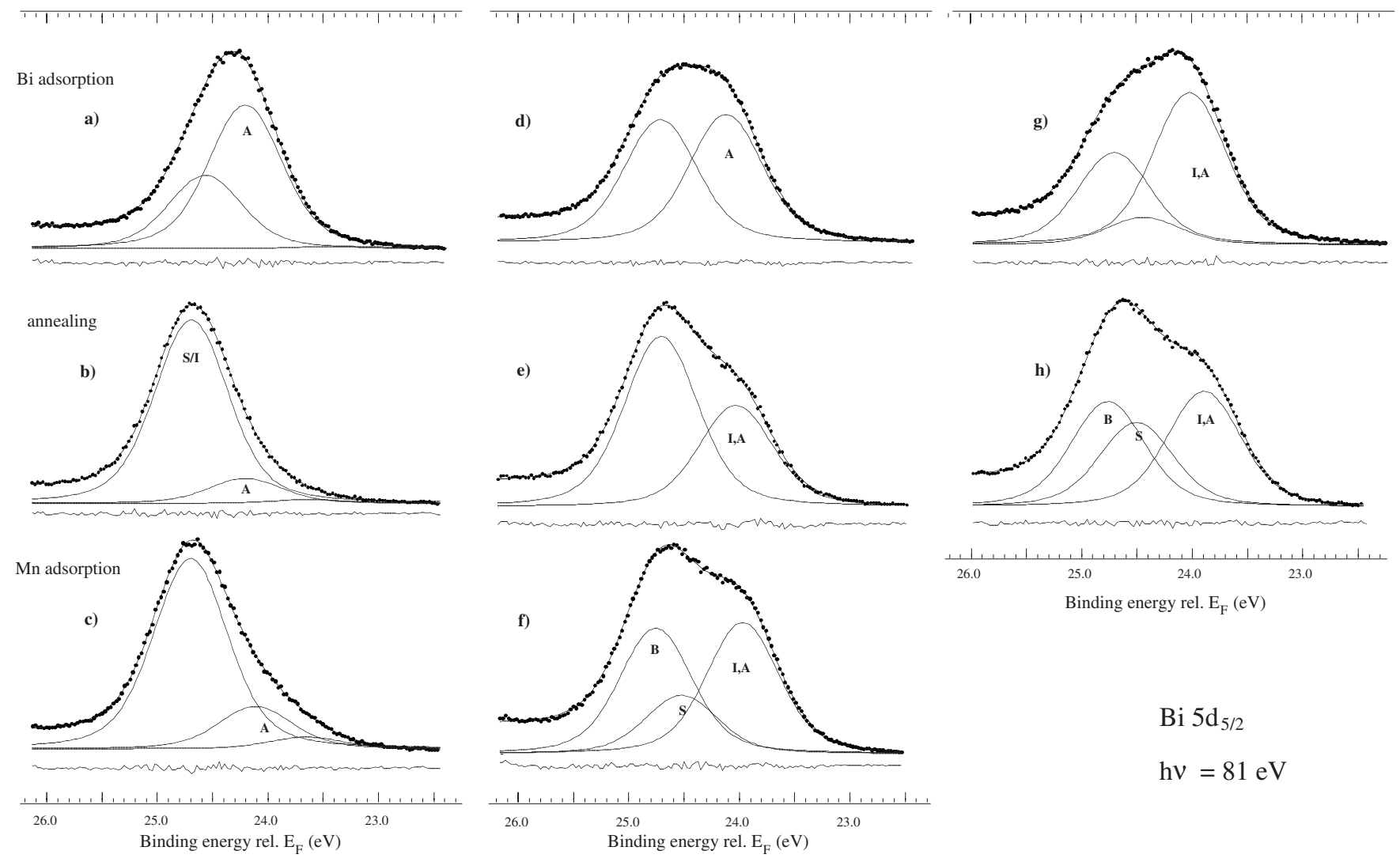

FIG. 4. Normal emission Bi5 $\mathrm{d}_{5 / 2}$ spectra during growth progress. First row represents Bi deposited at room temperature, second row after annealing, and final row after Mn deposition at $200{ }^{\circ} \mathrm{C}$. Each column represents a Bi and Mn deposition cycle.

between components $\mathrm{B}$ and $\mathrm{A}(1.0 \mathrm{eV}$ vs $0.87 \mathrm{eV})$, indicating a larger binding energy of the adsorbed species on the MnAs-terminated surface. The energy separation B-S1 is very similar on the as-grown and MnAs-terminated surfaces (around $0.30 \mathrm{eV}$ ) while B-S2 is again significantly larger for the MnAs-terminated surface $(0.76 \mathrm{eV}$ vs $0.45 \mathrm{eV})$.

With adsorption of the first monolayer $\mathrm{Bi}$ some changes are observed in the As3d spectrum. Apart from an overall intensity reduction, seen in Fig. 2, the relative intensities of the different components are affected. The most apparent change is the reduction in component A, i.e., that reflecting adsorbed As. However, as it is not completely removed, we conclude that some As is floating on top of the Bi-covered surface. This is most likely unintentionally adsorbed As in the growth-chamber atmosphere. Furthermore, we note that components $\mathrm{S} 1$ and $\mathrm{S} 2$ also remain, confirming the presence of As on the surface. The intensity of $\mathrm{S} 1$ is actually increased relative $\mathrm{B}$, which is of course mainly a consequence of attenuation of $\mathrm{B}$ by the $\mathrm{Bi}$ overlayer. The most significant observation is that the intensity of $\mathrm{X}$ is practically unchanged. This component clearly reflects atoms in a stable bonding state, supporting our earlier assignment as As atoms in the MnAs layer. We conclude that the adsorbed $\mathrm{Bi}$ atoms bind to As in the MnAs layer, as already inferred from the workfunction data. The adsorption of Bi results in a slight shift of the $\mathrm{X}$ component toward higher binding energy as indicated in Fig. 3.

After deposition of $2 \mathrm{ML} \mathrm{MnBi}$ an intensity reduction is observed in the As3d emission (cf. Fig. 2). The main change in the component-resolved analysis spectrum is a complete removal of component A. S1 and S2 are still present, though S2 with even lower intensity, showing that some As is still present on the surface. The MnAs-related component X remains with approximately unchanged relative intensity, the small reduction (about 10\%) relative the preceding spectrum may be due to diffraction effects or even an artifact in the fitting procedure.

The fact that no new component is found in the As3d spectra from the MnBi covered samples could be taken as an indication of no significant As intermixing in the MnBi layer. However, this observation alone cannot be taken as a safe argument for excluding intermixed As, since the corresponding spectral component could coincide with one of the components present prior to $\mathrm{MnBi}$ deposition. If As is intermixed in $\mathrm{MnBi}$, it is reasonable to expect that the corresponding emission ought to be close to that from MnAs, i.e., the component labeled X. Since the relative intensity of this component remains rather unaffected by the presence of $\mathrm{MnBi}$, it is clear that any additional contribution from intermixed As must be very small, significantly smaller than that of any other individual component in the As3d spectrum. By comparing this with the intensity of the "bulk" component in the Bi spectra (see below), and assuming similar excitation cross sections for As and $\mathrm{Bi}$ at $81 \mathrm{eV}$ photon energy, ${ }^{17}$ we must conclude that the concentration of $\mathrm{As}$ in the $\mathrm{MnBi}$ overlayer is at most in the range of $1 \%$.

We next turn to the apparently less complex Bi5d emission. Spectra obtained at different stages of treatment are 
shown in Fig. 4. The spectra are normalized to approximately the same amplitude and the energy is given relative the Fermi energy, which is assumed to be unaffected by the surface treatments. Directly after RT adsorption [spectrum (a)] two components are found, separated by $0.36 \mathrm{eV}$. The presence of two components is tentatively ascribed to $\mathrm{Bi}$ adsorbed on the As-terminated surface in different configurations. $\mathrm{Bi}$ adsorbed in the subsequent steps on $\mathrm{Mn}$ terminated surfaces was characterized by a single Bi component (see below). Upon annealing the intensity is clearly redistributed. Again two components are found, separated in energy by $0.49 \mathrm{eV}$. This is clearly larger than before annealing and shows that some reaction has taken place. The smaller component (A) coincides with the main one in the previous spectrum, and is thus ascribed to adsorbed, unreacted $\mathrm{Bi}$. Its presence shows that the $\mathrm{Bi}$ dosing was somewhat higher than intended. The main component is consequently associated with reacted $\mathrm{Bi}$, i.e., atoms bonding to As in the surface layer. In the subsequent step the sample was covered with 1.0 ML Mn [the Mn flux was calibrated by means of RHEED oscillations during growth of the underlying (GaMn)As]. This treatment leads to some intensity reduction but leaves otherwise the main component unaffected. The spectrum tails somewhat on the high-energy side, which can be accounted for by introducing a third Bi component. As this component does not appear in any later spectra, we are not able to identify its origin. The relative increase in component A suggests that the excess $\mathrm{Bi}$ is floating on top of the Mn layer. This is supported by the development with further Bi adsorption. We see in spectrum (d) that the intensity of component A is enhanced after RT deposition of another Bi layer and under the more surface sensitive conditions at $60^{\circ}$ emission angle (not shown) this component is clearly dominating.

Annealing of this surface results in spectrum (e). As expected, the adsorbed component is reduced, but in comparison with spectrum (b), which was obtained after equivalent annealing, spectrum (e) contains an additional component on the high-energy side. Considering the preparation history $\mathrm{Bi}$ should occur in at least two inequivalent sites: (i) atoms from the first deposition should be mainly located between the last substrate As layer and the Mn layer deposited in step (c), i.e., interface sites, and (ii) $\mathrm{Bi}$ atoms from the second adsorption cycle, now forming the surface layer reacted with Mn. It is natural to expect the surface sites to dominate, so we ascribe the largest of the two peaks to these sites. We also note that the smaller of the two components is slightly shifted (by about $80 \mathrm{meV}$ ) relative component $\mathrm{A}$ in the previous spectrum. Another observation is that the spectrum recorded at $60^{\circ}$ emission angle shows only a slight change in the relative intensity of the two components. These observations, along with the subsequent development with a new Mn adsorption, strongly suggest that the smaller of the two components in spectrum (e) actually contains contributions from the interface atoms as well as atoms in an adsorbed state due to excess $\mathrm{Bi}$. Such an arrangement would tend to compensate the expected angular dependence.

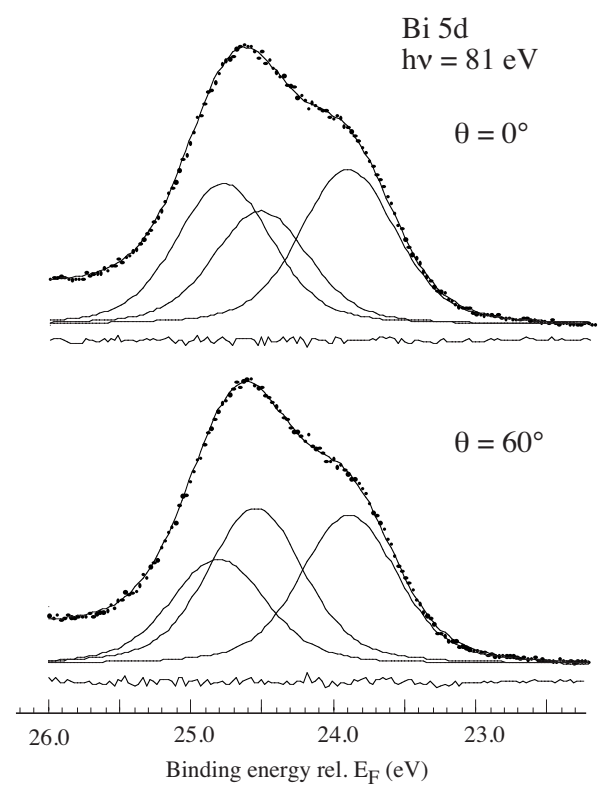

FIG. 5. Bi5 $\mathrm{d}_{5 / 2}$ in normal and $60^{\circ}$ emission angle after third Bi deposition and annealing. The angular dependence clearly supports our assignment of the surface component.

Following the second Mn adsorption [spectrum (f)], we see that the total width is further increased somewhat. The decomposition reveals the appearance of a third component, which is not expected considering that no new $\mathrm{Bi}$ has been supplied. We can explain the new component by assuming, as above, that some excess $\mathrm{Bi}$ above one monolayer was added in the latest deposition steps. By adding $\mathrm{Mn}$, we thus not only transform the previous surface $\mathrm{Bi}$ into sites sandwiched between two Mn planes (B) but have in addition a new partial surface layer of reacted $\mathrm{Bi}$ (since the Mn adsorption was done at $200{ }^{\circ} \mathrm{C}$ ) on top of the last Mn layer. The identification of the new surface component $\mathrm{S}$ is based on the fact that this component is enhanced relative the others at off-normal emission. We also note that the other two components (B and I) appear with similar intensities also in offnormal emission. As above, we ascribe this to the coinciding emission from interface $\mathrm{Bi}$ and unreacted $\mathrm{Bi}$ floating on the surface.

The third RT adsorption of Bi resulted again in increased emission on the high-energy side of the spectrum at the location of unreacted adsorbed $\mathrm{Bi}$. The $\mathrm{S}$ component is reduced clearly more than $\mathrm{B}$, which we believe is due to partial incorporation of the surface $\mathrm{Bi}$ into the adsorbed unreacted layer. However, after new annealing [spectrum (h)] the surface component is significantly enhanced and the spectrum contains three components as described above. The angular dependence (Fig. 5) supports the assignment of the surface component but also shows that component A/I (emission from adsorbed and interface $\mathrm{Bi}$ ) is relatively strong in large emission angles. It is clear that the conversion from adsorbed to reacted surface $\mathrm{Bi}$ is incomplete under the present conditions. 


\section{SUMMARY}

We have demonstrated that MnAs-terminated (GaMn)As layers can be overgrown with at least two monolayers $\mathrm{MnBi}$ using alternating $\mathrm{Bi}$ and $\mathrm{Mn}$ deposition and annealing. The
MnBi layer maintains the same $1 \times 2$ surface reconstruction as the GaMnAs surface and is apparently coherent with the underlying substrate. It seems, however, that incorporation of the third adsorbed Bi layer into the developing MnBi overlayer is hampered, probably as a result of lattice mismatch.
${ }^{1}$ S. H. Chun, S. J. Potashnik, K. C. Ku, P. Schiffer, and N. Samarth, Phys. Rev. B 66, 100408(R) (2002).

${ }^{2}$ C. Rüster, C. Gould, T. Jungwirth, J. Sinova, G. M. Schott, R. Giraud, K. Brunner, G. Schmidt, and L. W. Molenkamp, Phys. Rev. Lett. 94, 027203 (2005).

${ }^{3}$ M. Adell, V. Stanciu, J. Kanski, L. Ilver, J. Sadowski, J. Z. Domagala, P. Svedlindh, F. Terki, C. Hernandez, and S. Charar, Appl. Phys. Lett. 86, 112501 (2005).

${ }^{4}$ M. Wang, R. P. Campion, A. W. Rushforth, K. W. Edmonds, C. T. Foxon, and B. L. Gallagher, Appl. Phys. Lett. 93, 132103 (2008).

${ }^{5}$ T. Jungwirth, K. Y. Wang, J. Mašek, K. W. Edmonds, J. König, J. Sinova, M. Polini, N. A. Goncharuk, A. H. MacDonald, M. Sawicki, A. W. Rushforth, R. P. Campion, L. X. Zhao, C. T. Foxon, and B. L. Gallagher, Phys. Rev. B 72, 165204 (2005).

${ }^{6}$ S. Ohya, K. Ohno, and M. Tanaka, Appl. Phys. Lett. 90, 112503 (2007).

${ }^{7}$ D. Chiba, Y. Nishitani, F. Matsukura, and H. Ohno, Appl. Phys. Lett. 90, 122503 (2007).
${ }^{8}$ Y. Q. Xu, B. G. Liu, and D. G. Pettifor, Phys. Rev. B 66, 184435 (2002).

${ }^{9}$ J. C. Zheng and J. W. Davenport, Phys. Rev. B 69, 144415 (2004).

${ }^{10}$ M. Adell, J. Adell, L. Ilver, J. Kanski, J. Sadowski, and J. Z. Domagala, Phys. Rev. B 75, 054415 (2007).

${ }^{11}$ U. Nowak, U. Rüdiger, P. Fumagalli, and G. Güntherodt, Phys. Rev. B 54, 13017 (1996), and references therein.

${ }^{12}$ M. Nakada and M. Okada, IEEE Trans. Magn. 30, 4431 (1994).

${ }^{13}$ M.-F. Li, T. Ariizumi, K. Koyanagi, and S. Suzuki, Jpn. J. Appl. Phys., Part 1 46, 3455 (2007).

${ }^{14}$ J. Sadowski, J. Domagala, J. Bak-Misiuk, S. K. M. Sawicki, K. Swiatek, J. Kanski, L. Ilver, and V. Ström, J. Vac. Sci. Technol. B 18, 1697 (2000).

${ }^{15}$ I. Ulfat, J. Adell, L. Ilver, and J. Kanski (unpublished).

${ }^{16}$ M. Adell, L. Ilver, J. Kanski, J. Sadowski, R. Mathieu, and V. Stanciu, Phys. Rev. B 70, 125204 (2004).

${ }^{17}$ J. J. Yeh and I. Lindau, At. Data Nucl. Data Tables 32, 1 (1985). 